In doing a post.mortem examination of this kind, one naturally looks first for the commoner causes of acute disease, and the appendix is early searched for on this account. Unfortunately, the moving of the intestines in the search for the appendix disturbed the position of the ileum, and the position of the upper limit of the volvulus must remain more or less hypothetical. The extreme distension affected about $20 \mathrm{in}$. of the ileum, and the upper limit of the volvulus was probably in this position. In recorded cases of volvulus of the ileum, both the upper and lower limits seem in some cases to have been more or less indefinite.

Death supervened in five hours in this case, and was undoubtedly due to shock following an acute strangulation of a comparatively large amount of bowel, and that bowel part of the small intestine which is of much more vital importance than the large. From the immense size this part of intestine reached, and the amount of congested blood in it, there was practically a severe haemorrhage into it, as far as the general circulation was concerned. This must have greatly increased the already severe shock from which the patient was suffering.

Shock is defined as a condition of exhaustion of the vasomotor centre. Crile has shown that if the abdomen of an animal is opened and the intestines exposed, a fall of blood pressure occurs. This is associated with a vascular dilatation of the splanchnic area, and a marked degree of shock occurs. The animal bleeds into its own portal system. Anaemia of the vasomotor centre results, and sets up a general vasomotor paralysis, which increases as the vasomotor centre becomes exhausted. Treves states that " the grave initial symptoms of intestinal obstruction do not depend upon the fact that the bowel is obstructed, but upon the severe injury to the sympathetic nerves involved." Crile's observations show that this injury acts by producing an inhibition of the vaso-constrictor nerves, and ultimate paralysis of these nerves by exhaustion of the vasomotor centre. The resulting dilatation of the vessels of the splanchnic area causes a great fall in the systemic blood pressure and coincident shock.

In the case of this man there was an added haemorrhage into the part of the intestine forming the volvulus, thus increasing the factors causing shock, and making it much more profound owing to the added anaemia of the brain.

I can find no record of death ensuing in so short a time from intestinal obstruction, although several cases have been recorded where death ensued within twenty-four hours. Dr. Bryant suggests that the man was one who did not complain much, and that he may have had some pain earlier than the time stated.

The part of the ileum involved in this case, the lower part, is most frequently the seat of volvulus when it occurs in the small intestine. Treves says "the majority of patients exhibiting this form of volvulus have been males with a mean age of 25 years." Cases of volvulus of the small intestine in cases of typhoid fever, simulating perforation, are mentioned by Roberts; the symptoms were severe abdominal pain and collapse. Eliot has recorded a case in which a volvulus of the ileum, associated with one of the pelvic colon, caused great shock, with a pulse of 140 , thready and very weak, coldness and lividity, and apathy, but the facial expression showed intense suffering. In the case I have recorded the symptoms of shock were present to such a degree as to cause death within a few hours.

\section{TWO CASES OF APPENDICITIS.}

\section{By H. GOODWYN, F.R.C.S.EDIN.,} BOVEY TRACEY.

The following two cases of appendicitis presenting some unusual features are, I think, worth recording.

$$
\text { CASE I. }
$$

Mrs. C., aged 36, had for a period of two months repeated attacks of severe appendicular colic, accompanied on each occasion by a rise of temperature- $101^{\circ}$ to $102^{\circ}$-with severe abdominal pain, referred at first to the umbilical region, and latterly localized to the region of the appendix, the maximum point of tenderness being over McBurney's point. These attacks subsided under hot fomentations, rest in bed, etc., but the attacks became more frequent and intervals shorter; also they were more severe in character. The patient was three months pregnant, but in the light of the increasing severity of the pain, and frequency of attacks, it was decided to operate, and at the worst the risk of miscarriage was less than the possibility of the formation of an abscess.

Operation.-The caecum was much bound down by adhesions, and the tip of the appendix was adherent to the pelvic brim. There was great difficulty in withdrawing the caecum sufficiently to expose the base of the appendix, and to free the appendix itself, owing to numerous adhesions, but eventually this was accomplished, and a long inflamed appendix was removed, and the stump invaginated by purse-string suture, and covered by Lembert's sutures.

The subsequent history was uneventful; on the next day temperature and pulse were normal, and on the fourteenth day the patient returned home; no signs of uterine disturbance had occurred, and she remained well after six weeks. On opening the appendix, a nematode worm about $1 \frac{1}{2}$ in. in length was found, and there was a haemorrhagic condition of the mucous. lining of the whole organ, and an ulcer commencing to form on the floor, which would in all probability have gone on very shortly to perforation. I think the result amply justified the possible risk of cutting short the pregnancy.

\section{CASE II.}

A child aged 4, who for about twelve months had had attacks nome very severe, of abdominal pain without localized tenderness. principally referred to the umbilical region. Six weeks before operation she had a severe attack of pain, with rise of temperature to $102^{\circ}$; vomiting and marked constipation. There was no tenderness over the appendix at first; in about four days the pain was localized. The appendix, which was much inflamed, $4 \frac{1}{3}$ in. long, and considerably swollen, was isolated without difficulty. It was treated by invagination with purse-string suture, and closed over with Lembert's sutures. The recovery was uneventful; there was no rise of temperature or pulse, and the stitches were removed on the eighth day.

On opening the appendix a haemorrhagic condition of the mucous lining was seen, and a small fishbone, about ${ }_{5}^{5}$ in. long. lying free in the lumen. There was also evidence of an old kink, as represented by a transverse band of scar tissue.

Both these cases are illustrative of the advantage of early operation when symptoms of colic recur without. obvious cause; in the second case, though the temperature: and pulse became normal in a few days, during the whole five weeks there was never complete freedom from slight. attacks of pain, sometimes lasting only a few minutes, norfrom tenderness on deep pressure over the appendix.

The technique I practised was by incision through the skin over the outer border of the rectus, which was pushed inwards without splitting its fibres. 1 think this method" has distinct advantages over the gridiron method, as especially in the first case it would have been nearly impossible to obtain sufficient room for the necessary manipulations without dividing the fibres of the internab oblique and transversalis. I followed Professor von Eiselsberg, of Vienna, in painting the skin with a spirit. lotion of iodine liniment both before the incision and after the stitches were introduced.

\section{A CASE OF VERY LARGE GALL BLADDER. SLCCESSFULLY TREATED BY EXCISION.*}

By F. W. COLLINSON, M.D.Edin., M.R.C.P.LOND.\% F.R.C.S.EDIN.

HONORARY MEDICAL:OFFICER, PRESTON ROYAL INFIRMARY.

THIs case is remariable owing to the immense size of the gall bladder, being amongst the largest on record.

Mrs. S., aged 31, was sent to me by Dr. Callanan of Longridge on October 14th, 1908, suffering from general abdominas distension and pain in the right side of the abdomen.

\section{History.}

There was nothing of note in the family history, as all were healthy; the mother had died in childbirth. The patient herself had always been in good health, though pale. She had two. children, now living and healthy, born six and three years ago, with one miscarriage in between them.

The history of the present affection appeared to date from August, 1907, when she had a fall, for soon after she began to vomit each day, and in December she remained in bed on that account. She distinctly said there was then no swelling of the abdomen. In January she observed a swelling of the lower part. of the abdomen towards the right side. On February 13th she: had greatly increased in size, and was somewhat emaciated and markedly jaundiced. She was tapped, and 25 pints of fluid was drawn off, and the fluid must have been similar to that removed afterwards at the operation. Dr. Callanan was able to "feel the liver for 2 in. or 3 in. below the ribs," but he also wrote, rather prophetically, "this may, of course, be the sac of

Notes read, and patient shown, to the Clinical Branch of the Preston Medico-Ethical Society, February 3rd, 1909 